\title{
不同生态系统水分利用效率指标在黄土高原半干 旱草地应用的适宜性评价
}

\author{
刘晓 1,2 戚超 1,2 间艺兰 1,2 袁国富 $1,2 *$
}

${ }^{1}$ 中国科学院地理科学与资源研究所生态系统网络观测与模拟重点实验室, 北京 $100101 ;{ }^{2}$ 中国科学院大学资源与环境学院, 北京 100049

\begin{abstract}
摘 要 对近些年提出的两种生态系统水分利用效率指标——内在水分利用效率 $(I W U E)$ 和固有水分利用效率 $(u W U E)$ 与经典 的水分利用效率 $(W U E)$ 指标在黄土高原典型半干旱草地生态系统的应用情况进行了比较, 主要分析了不同指标在小时、日和 年尺度上的一致性和稳定性, 并提出优化水分利用效率 $(o W U E)$ 指标, 比较了不同定义的水分利用效率指标在模拟总初级生 产力 $(G P P)$ 逐日动态过程上的表现。结果显示: IWUE和 $u W U E$ 适合在日和小时尺度分析植被的碳水耦合特征, WUE适合分析年 尺度的碳水耦合特征; oWUE指标在描述碳水耦合特征方面与 $u W U E$ 相比并没有本质性改善, 但在用于模拟 GPP动态过程时 模拟效果改善明显; 采用 $O W U E$ 和 $u W U E$ 指标能改善植被生长盛期的GPP动态过程模拟, 但在生长季早期和晚期的模拟效果 比WUE差。IWUE指标不适合模拟GPP动态过程。
\end{abstract}

关键词＼cjkstart水分利用效率; 内在水分利用效率; 固有水分利用效率; 优化水分利用效率; 总初级生产力模拟

引用格式: 刘晓, 戚超, 闻艺兰, 袁国富 (2017). 不同生态系统水分利用效率指标在黄土高原半干早草地应用的适宜性评价. 植物生态学报, 41, 497-505. doi: 10.17521/cjpe.2016.0378

\section{Revised algorithm of ecosystem water use efficiency for semi-arid steppe in the Loess Plateau of China}

LIU Xiao ${ }^{1,2}$, QI Chao ${ }^{1,2}$, YAN Yi-Lan ${ }^{1,2}$, and YUAN Guo-Fu ${ }^{1,2 *}$

${ }^{1}$ Key Laboratory of Ecosystem Network Observation and Modeling, Institute of Geographic Sciences and Natural Resources Research, Chinese Academy of Sciences, Beijing 100101, China; and ${ }^{2}$ College of Resources and Environment, University of Chinese Academy of Sciences, Beijing 100049, China

\begin{abstract}
Aims We evaluated the applicability of different measures of water use efficiency through analyzing the coupled dynamics of GPP and evapotranspiration in the semi-arid steppe in the Loess Plateau of China. Our objective is to explore the applicability of two quantitative measures of ecosystem water use efficiency-inherent water use efficiency (IWUE) and underlying water use efficiency $(u W U E)$ — for the semi-arid steppe and to endeavor necessary modifications.

Methods The consistency and stability of three indices of water use efficiency formulations (i.e. WUE, IWUE, $u W U E$ ) were calculated and compared at hourly, daily and annual time scales before proposing an optimal water use efficiency (oWUE). These indices were additionally used to quantify their importances in modeling the diel change of gross primary production (GPP). The yielded-accuracy of the prediction was used for justifying their uses.

Important findings $I W U E$ and $u W U E$ appeared suitable for examining the coupled water-carbon characteristics of vegetation at hourly and daily scales, whereas $W U E$ was more plausible on the annual and interannual scales. The optimized water use efficiency index did not improve the prediction of the coupled water-carbon characteristics as compared with $u W U E$, but it improved the prediction of GPP and its dynamics. $o W U E$ and $u W U E$ improved the predictions of $G P P$ in the peak growing period, while $W U E$ predicted the GPP better at the early and late growing season. Interestingly, we found that $I W U E$ was not suitable for predicting $G P P$ and its dynamics. The results will be of great importance in modeling the effects of climate change on the carbon assimilation and water cycle for the future.
\end{abstract}

Key words water use efficiency; inherent water use efficiency; underlying water use efficiency; optimal water use efficiency; predicted gross primary production

收稿日期Received: 2016-12-12 接受日期Accepted: 2017-02-28

* 通信作者Author for correspondence (E-mail: yuangf@igsnrr.ac.cn) 
Citation: Liu X, Qi C, Yan YL, Yuan GF (2017). Revised algorithm of ecosystem water use efficiency for semi-arid steppe in the Loess Plateau of China. Chinese Journal of Plant Ecology, 41, 497-505. doi: 10.17521/cjpe.2016.0378

碳同化和水分蒸腾由植物通过气孔行为控制, 通常用水分利用效率 $(W U E$, 即单位水分消耗量所 同化的碳量)定量表达 (Singh \& Gupta，1977; Baldocchi, 1994)。在生态系统水平上, $W U E$ 能够反 映植物的水分利用策略(胡中民等, 2009), 以及植物 在光合碳同化过程中获取的碳与消耗的水之间的权 衡, 即碳水耦合特征(Farquhar, 1977)。生态系统 WUE也是全球变化生态学研究的一个重要指标 (Scanlon \& Albertson, 2004), 可用来评估生态系统 对气候变化和大气 $\mathrm{CO}_{2}$ 浓度增加的动态响应( $\mathrm{Niu}$ et al., 2011)。经典的生态系统WUE为总初级生产力 $(G P P)$ 与地表蒸散(ET)的比值(Beer et al., 2009)。这 个定义通常适于在月、年等长时间尺度上分析生态 系统的碳水耦合特征及其对环境变化的响应。最近 的研究定义了两种新的水分利用效率: 内在水分利 用效率(IWUE) (Beer et al., 2009)和固有水分利用效 率( $u W U E$ ) (Zhou et al., 2014, 2015)。新的定义主要 考虑到饱和水汽压差 $(V P D)$ 在日尺度和小时尺度上 对碳水耦合过程的重要影响。Beer等(2009)将 $G P P \times$ $V P D$ 与 $E T$ 的比值称为 $I W U E$, 并利用全球不同生态 系统 43 个通量站的数据进行检验, 证实 $I W U E$ 较 $W U E$ 更适于在日尺度上分析生态系统碳水耦合机 制(Leonardi et al., 2012; Battipaglia et al., 2013; Grossiord et al., 2014)。Zhou等(2014, 2015)通过分析 植物碳水耦合过程的内在机制, 认为 $G P P \times V P D^{0.5}$ 与 $E T$ 的比值能更合理地描述植物碳水耦合特征, 且 能在小时尺度上使用, Zhou等 $(2014,2015)$ 将其称为 $u W U E$ 。事实上, $W U E 、 I W U E$ 和 $u W U E$ 这 3 个定义可 用一个通用的式子 $G P P \times V P D^{k} E T$ 统一起来, 其差 别仅是 $k$ 的取值不同: 当 $k=0$ 时, $G P P \times V P D^{k} / E T=$ $W U E$; 当 $k=1$ 时, $G P P \times V P D^{k} E T=I W U E$; 当 $k=0.5$ 时, $G P P \times V P D^{k} / E T=u W U E$ 。因此, 针对特定生态 系统, 可寻找其最优的 $k$ 值, 用于不同时间尺度的 生态系统碳水耦合特征分析(Zhou et al., 2014, 2015)。

水分利用效率指标可用于估算大时间尺度的 $G P P$ 和 $E T$ 。生态系统水分利用效率代表植物固有的 内在属性, $G P P$ 和 $E T$ 通常呈显著线性相关, 当获取 大尺度的 $G P P$ 或者 $E T$ 值后，可以基于水分利用效率 指标估算大时间尺度的 $E T$ 或 $G P P$ 值 (Beer et al., 2007; Yang et al., 2013)。建立适用于较短时间尺度
上应用的水分利用效率指标，能更好地提高 $G P P$ 模 拟精度, 尤其是对 $G P P$ 动态过程的模拟(Zhou et al., 2015)。

目前对上述3种指标的应用评价较少，提出合 理的水分利用效率指标, 不仅能更好地解释生态系 统碳水耦合特征的变化机制, 而且可应用于对生态 系统碳水循环过程的估算与模拟(王绍刚等, 2008; 张良侠等, 2014)。本研究结合中国科学院水利部水 土保持研究所神木侵蚀与环境试验站的通量数据, 分析比较了上述3种水分利用效率在黄土高原典型 草地生态系统中应用的适宜性和存在的问题：(1)评 价3种指标在小时、日和年尺度上的表现; (2)分析是 否存在适合黄土高原草地生态系统的优化水分利用 效率分析指标, 即是否存在最优 $k$ 值 $\left(k^{*}\right)$; (3) 分析不 同定义水分利用效率指标在模拟 $G P P$ 日动态中的精 度和存在的问题。本研究通过分析和比较这3种生态 系统水分效率指标在反映生态系统碳水耦合特征上 的表现，以及可能的应用途径，为不同的水分利用 效率指标在黄土高原半干旱草地生态系统的应用提 供借鉴。

\section{1 材料和方法}

\section{1 研究区概况}

研究地点位于黄土高原六道沟流域中国科学 院水利部水土保持研究所神木侵蚀与环境试验站 $\left(38.79^{\circ} \mathrm{N}, 110.37^{\circ} \mathrm{E}\right.$ ，海拔1 $\left.256 \mathrm{~m}\right)$ 。该区域属温带 大陆性半干旱气候, 年平均气温7-9 ${ }^{\circ} \mathrm{C}$, 年降水量 $437.4 \mathrm{~mm}$, 年内降水分布不均, 6-9月降水量可占全 年降水量的 $70 \%-80 \%$ 。实验地为撂荒地自然演替形 成的半干旱草地植被。优势种为长芒草 (Stipa bungeana)和赖草(Leymus secalinus), 同时伴生有草 木樨状黄耆(Astragalus melilotoides)、茵陈蒿(Artemisia capillaris)和兴安胡枝子(Lespedeza davurica) 等。 绿色植被平均盖度为 $72 \%$, 但由于常年有枯草覆盖, 几乎没有裸露土壤。研究区土壤为典型的黄土(赵纯 等, 2015)。研究区植被为黄土高原半干旱区的典型 自然植被，在黄土高原半干旱区具有代表性。

\section{2 数据获取和处理}

本研究采用开路式浴度相关系统(EC)观测研究 区典型半干旱草地生态系统碳水通量特征。浴度相 
关系统由三维超声风速仪(CSAT-3, Campbell, Logan, USA) 和 $\mathrm{CO}_{2} / \mathrm{H}_{2} \mathrm{O}$ 快速响应红外分析仪 (LI7500, Li-COR, Lincoln, USA)等组成, 主要用于测定地表 与大气间 $\mathrm{CO}_{2}$ 与水汽通量交换, 观测高度为 $2 \mathrm{~m}$ 。仪 器采样频率为 $10 \mathrm{~Hz}$ 。对常规气象要素如净辐射 $\left(R_{\mathrm{n}}\right)$ 、空气温度 $(T)$ 、空气相对湿度 $(R H)$ 、风速 $(W)$ 、 降水量 $(P)$ 等也进行观测。同时，在地表下5、10、

20、30和50 $\mathrm{cm}$ 土层处安装探头, 测定土壤含水量 $(S W)$ 、土壤热通量 $(G)$ 和土壤温度 $\left(T_{\mathrm{S}}\right)$ 等指标。

利用软件Eddypro对原始数据进行处理, 将其 转换为 $0.5 \mathrm{~h}$ 通量数据。由于降水、断电、仪器损坏 等造成一些数据的缺失及异常, 需进行数据插补。 其中, 对白天的生态系统碳交换量 $(N E E)$, 主要基 于Michaelis-Menten公式进行插补(于贵瑞等, 2010):

$$
N E E=\frac{\alpha P P F D \beta}{\alpha P P F D+\beta}-R_{\mathrm{e}}
$$

式中, $P P F D$ 为入射到叶片上的光合有效光量子通 量密度 $\left(\mu \mathrm{mol} \cdot \mathrm{m}^{-2} \cdot \mathrm{s}^{-1}\right), \alpha$ 为表征光合作用最大光能转 化率的表观初始量子效率, $\beta$ 为光饱和时的表观最大 光合速率 $\left(\mu \mathrm{mol} \cdot \mathrm{m}^{-2} \cdot \mathrm{s}^{-1}\right), R_{\mathrm{e}}$ 为生态系统呼吸速率 $\left(\mu \mathrm{mol} \cdot \mathrm{m}^{-2} \cdot \mathrm{s}^{-1}\right)$ 。植物在不同生育期具有不同的 $\alpha 、 \beta$ 值, 为保证数据的准确性, $\alpha 、 \beta$ 值为逐月数据拟合 确定。

涡度相关系统测得 $\mathrm{CO}_{2}$ 通量即为 $N E E$ 值, 利用 如下公式将 $N E E$ 值转化为 $G P P$ 值:

$$
G P P=N E E+R_{\mathrm{e}}
$$

在夜间, $N E E$ 即为生态系统呼吸, 采用温度-水 分连乘公式插补:

$$
\begin{aligned}
& R_{\mathrm{e}}=R_{\mathrm{ref}} f(T) f\left(S_{\mathrm{w}}\right) \\
& f(T)=e^{E_{0}\left(\frac{1}{T_{\mathrm{ref}}-T_{0}}-\frac{1}{T_{\text {soil }}-T_{0}}\right)} \\
& f\left(S_{\mathrm{w}}\right)=e^{\left(b S_{\mathrm{w}}+c S_{\mathrm{w}}^{2}\right)}
\end{aligned}
$$

式中, $R_{\mathrm{ref}}$ 为参考温度 $\left(T_{\mathrm{ref}}\right)$ 下的生态系统呼吸 $\left(\mathrm{mg} \cdot \mathrm{m}^{-2} \cdot \mathrm{s}^{-1}\right), T_{\mathrm{ref}}$ 为参考温度 $\left(\right.$ 取 $15{ }^{\circ} \mathrm{C}$, 即 $288.15 \mathrm{~K}$ ), $E_{0}$ 为活动能量 (取 $\left.309 \mathrm{~K}\right), T_{\text {soil }}$ 为土壤温度 $(\mathrm{K})$, 本研
究取 $5 \mathrm{~cm}$ 深处的土壤温度, $T_{0}$ 为生态系统呼吸为 0 时 $5 \mathrm{~cm}$ 深处的土壤温度 $(\mathrm{K}), S_{\mathrm{w}}$ 为土壤 $5 \mathrm{~cm}$ 深处的含水 量 $\left(\mathrm{m}^{3} \cdot \mathrm{m}^{-3}\right), b 、 c$ 为试验参数, 根据植物生长季数据 逐年测得。

本研究中浴度相关数据的全天能量闭合度约 为 $64 \%$, 通过强制能量平衡获得实际 $E T$, 未闭合部 分的能量以波文比按比例分配给感热 $(H)$ 和潜热 $(L E)$ (Wohlfahrt et al., 2009)。

对于小范围 $E T$ 数据空缺 $(<2 \mathrm{~h})$, 采用线性内插 法。对于大于 $2 \mathrm{~h}$ 的 $E T$ 数据缺失, 通过采用参考蒸散 量 $E T_{0}$ 与实际蒸散量 $E T$ 的线性关系进行插补 (Priestley \& Taylor, 1972)。

通过数据处理和插补后, 能够形成完整的 $0.5 \mathrm{~h}$ 通量数据, 基于这些数据, 应用不同水分利用效率 指标在不同时间尺度的计算公式, 计算水分利用效 率指标值, 对全生长季数据进行对比, 剔除由于降 水导致的 $V P D$ 偏小的日尺度数据。年尺度的水分利 用效率计算仅考虑了生长季数据, 非生长季数据不 予考虑。

\section{3 不同水分利用效率定义的计算和分析方法}

Zhou等(2015)将WUE、IWUE和 $u W U E$ 通过下面 这个公式统一起来:

$$
W U E_{k}=G P P \times V P D^{k} / E T
$$

当 $k=0$ 时, $W U E_{k}$ 为 $W U E, k=1$ 时, $W U E_{k}$ 为 $I W U E, k=$ 0.5 时, $W U E_{k}$ 为 $u W U E$ 。在该公式中, 可能存在一个 最优的 $k$ 值 $\left(k^{*}\right)$, 使得 $G P P \times V P D^{k}$ 和 $E T$ 之间的相关 性最好, 将其定义为“优化水分利用效率 $(o W U E)$, , 分别通过下标 $0 、 1.0 、 0.5$ 和 $k^{*}$ 将上述 4 个水分利用效 率指标区分开来。表 1 归纳了 4 个水分利用效率指标 的符号、计算公式和单位。

利用 $G P P \times V P D^{k}(k=0 、 1.0 、 0.5)$ 与 $E T$ 线性相 关性来衡量不同水分利用效率指标在不同时间尺度 的表现, 相关系数 $(r$ )越高, 该指标越能表达生态系 统的碳水耦合特征(Zhou et al., 2014, 2015)。另外, 我们计算了 $W U E_{k}$ 的变异系数 $(C v$, coefficient of

表1 不同生态系统水分利用效率指标的定义与单位

Table 1 Basic information for various algorithms of calculating water use efficiency at ecosystem level

\begin{tabular}{llll}
\hline 类型 Type & 符号 Symbol & 代号 Code & 表达式 Formulation \\
\hline 水分利用效率 Water use efficiency & $W U E$ & $W U E_{0}$ & $G P P / E T$ \\
内在水分利用效率 Inherent water use efficiency & $I W U E$ & $W U E_{1.0}$ & $G P P \times V P D / E T$ \\
固有水分利用效率 Underlying water use efficiency & $u W U E$ & $W U E_{0.5}$ & $G P P \times V P D^{0.5} / E T$ \\
优化水分利用效率 Optimal water use efficiency & $o W U E$ & $W U E_{k^{*}}$ & $G P P \times V P D^{k^{*}} / E T$ \\
\hline
\end{tabular}


表2 不同水分利用效率指标在小时、日和年尺度的计算方法

Table 2 Algorithms for calculating water use efficiency (WUE) at hourly, daily and yearly time scale

\begin{tabular}{llll}
\hline $\begin{array}{l}\text { 时间尺度 } \\
\text { Time scales }\end{array}$ & $\begin{array}{l}\text { 水分利用 } \\
\text { 效率 } W U E\end{array}$ & $\begin{array}{l}\text { 内在水分利 } \\
\text { 用效率 } I W U E\end{array}$ & $\begin{array}{l}\text { 固有水分利 } \\
\text { 用效率 } u W U E\end{array}$ \\
$\begin{array}{l}\text { 小时尺度 } \\
\text { Hourly time scales }\end{array}$ & $\frac{G P P_{\mathrm{i}}}{E T_{\mathrm{i}}}$ & $\frac{G P P_{\mathrm{i}} \times V P D}{E T_{\mathrm{i}}}$ & $\frac{G P P_{\mathrm{i}} \times V P D^{0.5}}{E T_{\mathrm{i}}}$ \\
$\begin{array}{l}\text { 日尺度 } \\
\text { Daily time scales }\end{array}$ & $\frac{G P P_{\mathrm{d}}}{E T_{\mathrm{d}}}$ & $\frac{G P P_{\mathrm{d}} \times \overline{V P D_{\mathrm{d}}}}{E T_{\mathrm{d}}}$ & $\frac{G P P_{\mathrm{d}} \times\left(\overline{V P D_{\mathrm{d}}}\right)^{0.5}}{E T_{\mathrm{d}}}$ \\
$\begin{array}{l}\text { 年尺度 } \\
\text { Yearly time scales }\end{array}$ & $\frac{\sum G P P_{\mathrm{d}}}{\sum E T_{\mathrm{d}}}$ & $\frac{\sum\left(G P P_{\mathrm{d}} \times \overline{V P D_{\mathrm{d}}}\right)}{\sum E T_{\mathrm{d}}}$ & $\frac{\sum\left[G P P_{\mathrm{d}} \times\left(\overline{V P D_{\mathrm{d}}}\right)^{0.5}\right]}{\sum E T_{\mathrm{d}}}$ \\
\hline
\end{tabular}

$G P P_{\mathrm{i}}$ 和 $G P P_{\mathrm{d}}$, 小时尺度和日尺度总初级生产力; $E T_{\mathrm{i}}$ 和 $E T_{\mathrm{d}}$, 小时尺度和 日尺度蒸散量; $V P D$ 和 $\overline{V P D_{d}}$, 小时尺度和日尺度饱和水汽压差。

$G P P_{\mathrm{i}}$ and $G P P_{\mathrm{d}}$, gross primary productivity on hourly and daily time scales; $E T_{\mathrm{i}}$ and $E T_{\mathrm{d}}$, evapotranspiration on hourly and daily time scales; $V P D$ and $\overline{V P D_{d}}$, vapor pressure deficit on hourly and daily time scales. IWUE, inherent water use efficiency; $u W U E$, underlying water use efficiency.

variation, 标准差与平均值的比值), 来描述指标的 离散程度, $C v$ 值越小, 则该指标随时间的变化越小, 稳定性越高, 意味着指标的利用价值越高。

不同尺度的水分利用效率的计算方法见表2。 为方便描述, $W U E_{k, \mathrm{i}} 、 W U E_{k, \mathrm{~d}}$ 和 $W U E_{k, \mathrm{y}}$ 分别代表小 时、日和年尺度的 $W U E_{k}\left(k=0 、 1.0 、 0.5 、 k^{*}\right)$ 值。 $W U E_{k^{*}}$ 的算法与 $W U E_{0.5}$ 类似, 将 0.5 替换为 $k^{*}$ 即可。

公式(6)可通过寻找 $G P P \times V P D^{k}$ 与 $E T$ 之间的最 优线性相关性来获取 $k^{*}$ 。通过分析 $G P P \times V P D^{k}$ 与 $E T$ 的相关性, 以步长 0.001 调整 $k$ 值大小以获得 $k^{*}$ 值。本 文分析和比较了小时和日尺度的 $k^{*}$ 值。

为进一步分析不同水分利用效率指标在预测 $G P P$ 动态变化精度上的表现, 我们针对每一种水分 利用效率指标, 对日 $G P P$ 动态过程进行了模拟:

$$
G P P_{\mathrm{d}, \mathrm{m}}=E T_{\mathrm{d}} \cdot W U E_{k, \mathrm{y}} / V P D_{\mathrm{d}}^{k}
$$

式中, $G P P_{\mathrm{d}, \mathrm{m}}$ 为模拟的日 $G P P ; E T_{\mathrm{d}}$ 为日蒸散量 $(\mathrm{mm})$, 为涡度相关观测值; $V P D_{\mathrm{d}}$ 为日均饱和水汽压差 $(\mathrm{hPa})$; $W U E_{k, y}$ 为本研究获得的不同定义的年水分利用效 率指标年尺度值, $k$ 代表不同的水分利用效率指标, 为 $0 、 1.0 、 0.5$ 和 $k^{*}$ 。模拟精度通过比较模拟的 $G P P$ 与涡度相关观测获得的实测 $G P P$, 计算纳什效率系 数(NSE, Nash-Sutcliffe efficiency coefficient)来衡量, $N S E$ 值越接近 1, 表明模拟效果越好 (Legates \& McCabe, 1999)。

\section{2 结果和分析}

\section{1 不同水分利用效率指标在不同时间尺度上的 表现}

图1和图2分别显示了2014-2016年生长季小时
与日尺度的 $G P P 、 G P P \times V P D 、 G P P \times V P D^{0.5}$ 与 $E T$ 之间的关系，表3列出了不同水分利用效率指标的 相关统计数据，包括 $r 、 C v$ 、日均水分利用效率和年 尺度的水分利用效率。同时从图1和图2可以看出, 在小时和日尺度上, 连续 3 年的数据均显示 $G P P$ 与 $E T$ 的相关关系不如 $G P P \times V P D 、 G P P \times V P D^{0.5}$ 与 $E T$ 紧密, 同时 $G P P \times V P D^{0.5}$ 与 $E T$ 的相关关系比 $G P P \times$ $V P D$ 与 $E T$ 的相关关系紧密。这个结果与Zhou在北美 不同生态系统中的分析结果(Zhou et al., 2015)一致, 说明考虑 $V P D$ 对 $E T$ 的非线性影响, 能较好地定义日 和小时尺度的水分利用效率特征, 正如Zhou (2014) 所说, 在生态系统水平的较小时间尺度(小时与日 尺度)上, $u W U E$ 更能表明碳水通量之间的联系。

比较日尺度的 $C v$ 得出: $u W U E<W U E<I W U E$, 表明 $u W U E$ 指标随时间的变化最稳定, WUE次之, 而IWUE的稳定性最差。这个结果与 $r$ 显示的结果不 一致, 在相关系数方面, IWUE表现好于 $W U E$ 。相关 系数描述了植被碳水耦合的内在属性, 而变异系数 反映了植被碳水交换随时间的变化幅度。IWUE的变 异系数在 3 个指标中最高, 意味着该指标随时间变 化幅度最大。由于IWUE的结果受VPD变化的影响, 我们猜测研究区特殊的半干旱大陆型季风气候特征 可能是导致这一原因的主要因素。在大陆型季风气 候下, 半干旱区空气水汽的日变化较大, 影响了 $I W U E$ 的稳定性。而 $u W U E$ 的计算对 $V P D$ 进行了开方, 降低了VPD波动对指标值的影响。

日均水分利用效率与年尺度水分利用效率总 体上相差不大(表3), 体现了计算获得的年尺度水分 利用效率指标的合理性。但是不同指标的年际变化 相差非常大, 其中 $W U E$ 的年际变化最小 (41.6\%), uWUE次之 (85.8\%), IWUE的年际变化最大(130.3\%), 在这3年中, 年际变幅超过 1 倍。对照WUE的年际变 化幅度, 研究区 $U W U E$ 和IWUE在反映水分利用效率 的年际变化时放大了生态系统水分利用效率的年 际变化。

\section{2 优化水分利用效率指标的 $\boldsymbol{k}^{*}$ 值}

2014-2016年小时及日尺度 $k^{*}$ 及 $G P P \times V P D^{k^{*}}$ 与 $E T$ 线性关系相关系数见表 $4 \circ k^{*}$ 的取值范围基本集中 在0.4左右, 在不同年份上下浮动不大。

在同一年的同一时间尺度, $G P P \times V P D^{k^{*}}$ 与 $E T$ 的相关系数和 $G P P \times V P D^{0.5}$ 与 $E T$ 的相关系数相差小 于 0.02 , 尽管获取的 $k^{*}$ 值与 $u W U E$ 的 $k$ 取值 0.5 之间相

www.plant-ecology.com 

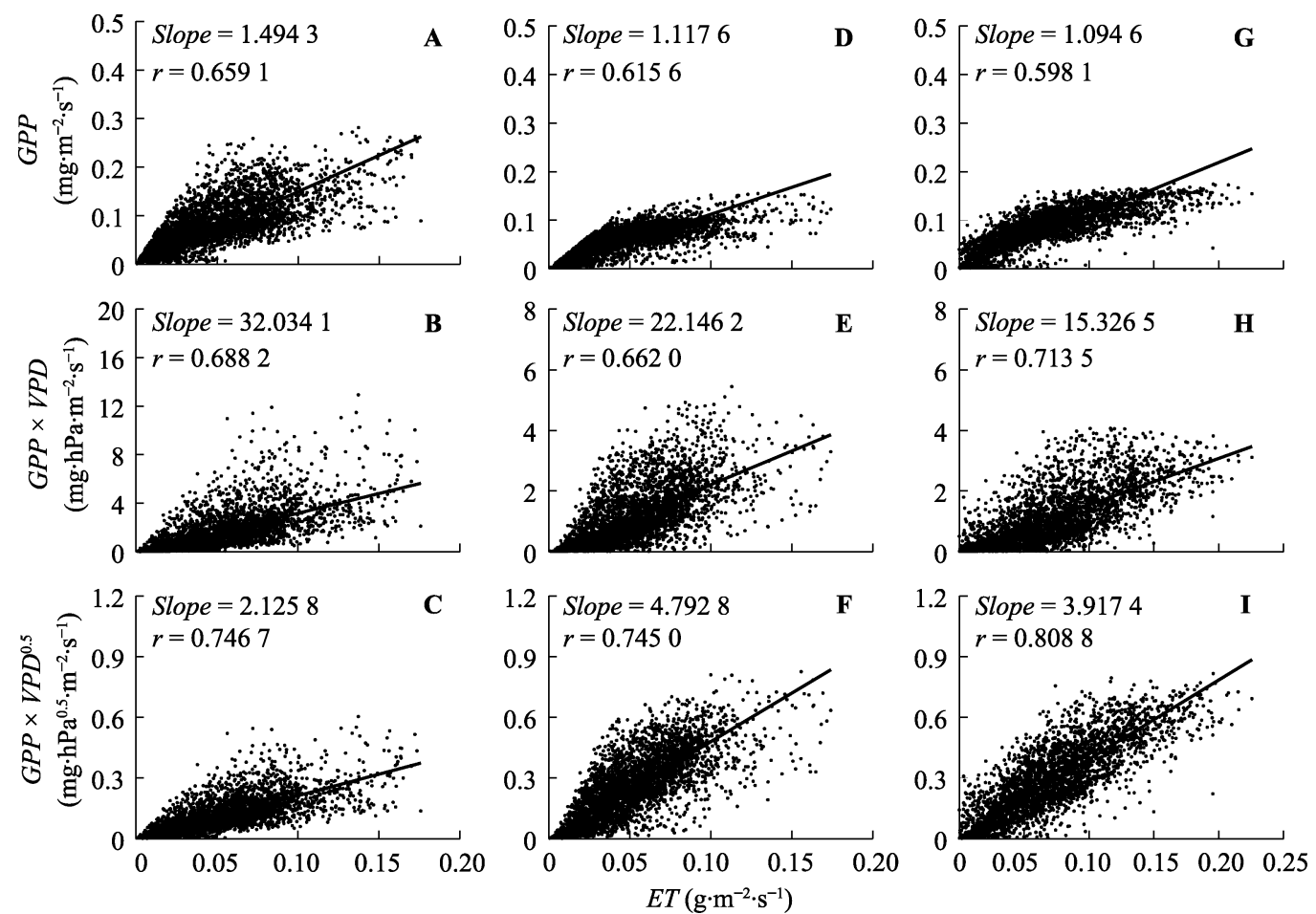

图1 2014-2016年生长季 $0.5 \mathrm{~h}$ 尺度 $G P P(\mathbf{A}, \mathbf{D}, \mathbf{G}) 、 G P P \times V P D(\mathbf{B}, \mathbf{E}, \mathbf{H}) 、 G P P \times V P D^{0.5}(\mathbf{C}, \mathbf{F}, \mathbf{I})$ 与 $E T$ 之间的关系。 $E T$, 蒸散 量; $G P P$, 总初级生产力; $V P D$, 饱和水汽压差。Slope, 斜率。

Fig. 1 Relationship between $G P P(\mathbf{A}, \mathbf{D}, \mathbf{G}), G P P \times V P D(\mathbf{B}, \mathbf{E}, \mathbf{H}), G P P \times V P D^{0.5}(\mathbf{C}, \mathbf{F}, \mathbf{I})$ and $E T$ at hourly scale during the growing season of 2014-2016. $E T$, evapotranspiration; $G P P$, gross primary productivity; $V P D$, vapor pressure deficit.
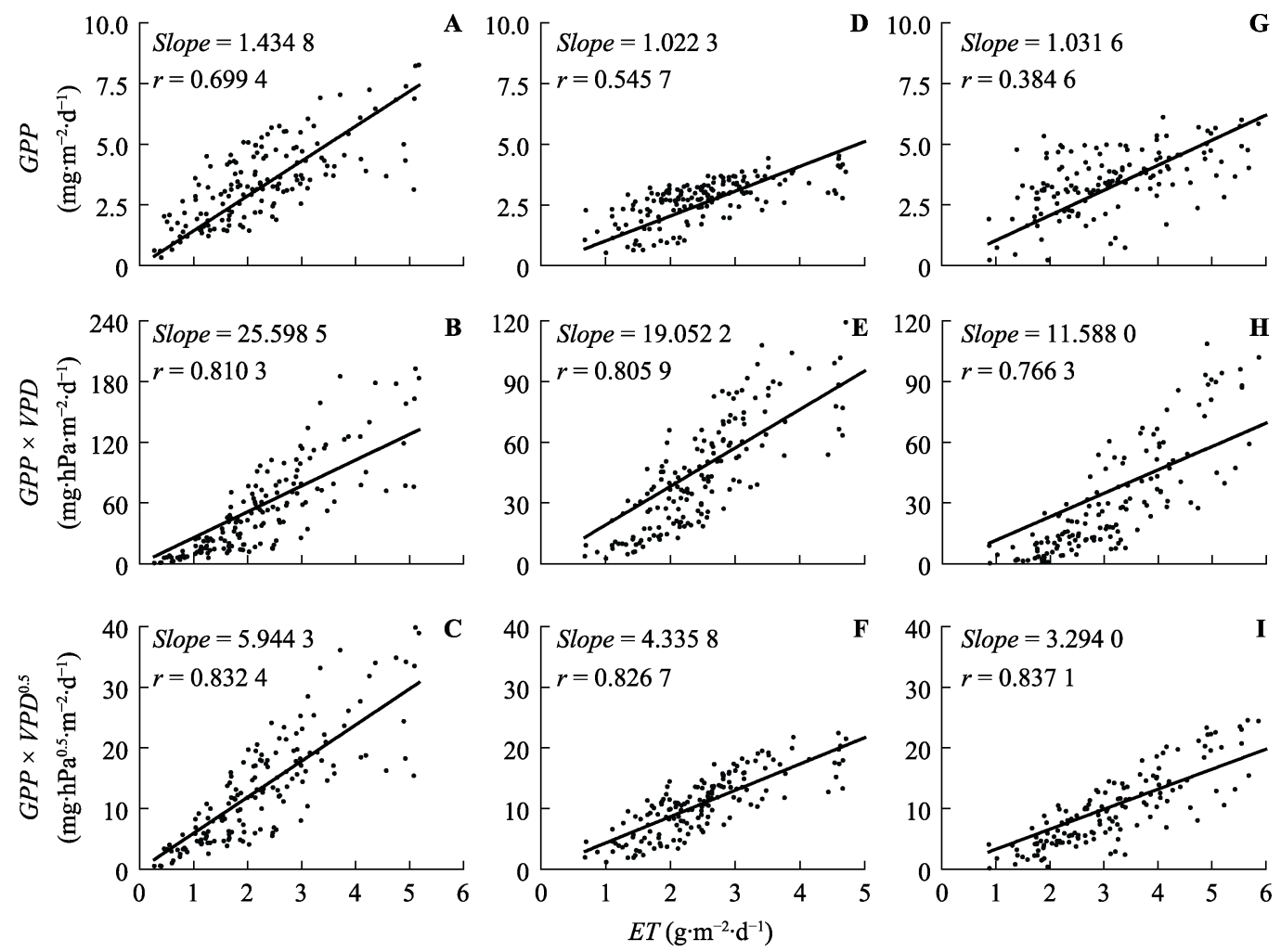

图2 2014-2016年生长季日尺度 $G P P(\mathbf{A}, \mathbf{D}, \mathbf{G}) 、 G P P \times V P D(\mathbf{B}, \mathbf{E}, \mathbf{H}) 、 G P P \times V P D^{0.5}(\mathbf{C}, \mathbf{F}, \mathbf{I})$ 与 $E T$ 之间的关系。 $E T$, 蒸散量; $G P P$, 总初级生产力; $V P D$, 饱和水汽压差。Slope, 斜率。

Fig. 2 Relationship between $G P P(\mathbf{A}, \mathbf{D}, \mathbf{G}), G P P \times V P D(\mathbf{B}, \mathbf{E}, \mathbf{H}), G P P \times V P D^{0.5}(\mathbf{C}, \mathbf{F}, \mathbf{I})$ and $E T$ at daily scale during the growing season of 2014-2016. $E T$, evapotranspiration; $G P P$, gross primary productivity; $V P D$, vapor pressure deficit. 
表3 2014-2016年日和年尺度水分利用效率 $(W U E)$ 、内在水分利用效率 $(I W U E)$ 和固有水分利用效率 $(u W U E)$ 变化比较

Table 3 Comparisons of the daily and yearly values of water use efficiency $(W U E)$, inherent water use efficiency $(I W U E)$, and underlying water use efficiency (uWUE) in 2014-2016

\begin{tabular}{|c|c|c|c|c|c|c|c|c|c|c|c|c|}
\hline \multirow{2}{*}{$\begin{array}{l}\text { 年份 } \\
\text { Year }\end{array}$} & \multicolumn{4}{|c|}{ 水分利用效率 $W U E$} & \multicolumn{4}{|c|}{ 内在水分利用效率 $I W U E$} & \multicolumn{4}{|c|}{ 固有水分利用效率 $u W U E$} \\
\hline & $\begin{array}{l}\text { 相关 } \\
\text { 系数 } \\
r\end{array}$ & $\begin{array}{l}\text { 变异 } \\
\text { 系数 } \\
C v\end{array}$ & $\begin{array}{l}\text { 日平均值 } \\
\text { Mean daily } \\
\text { value }\end{array}$ & $\begin{array}{l}\text { 年值 } \\
\text { Yearly } \\
\text { value }\end{array}$ & $\begin{array}{l}\text { 相关 } \\
\text { 系数 } \\
r\end{array}$ & $\begin{array}{l}\text { 变异 } \\
\text { 系数 } \\
C v\end{array}$ & $\begin{array}{l}\text { 日平均值 } \\
\text { Mean daily } \\
\text { value }\end{array}$ & $\begin{array}{l}\text { 年值 } \\
\text { Yearly } \\
\text { value }\end{array}$ & $\begin{array}{l}\text { 相关 } \\
\text { 系数 } \\
r\end{array}$ & $\begin{array}{l}\text { 变异 } \\
\text { 系数 } \\
C v\end{array}$ & $\begin{array}{l}\text { 日平均值 } \\
\text { Mean daily } \\
\text { value }\end{array}$ & $\begin{array}{l}\text { 年值 } \\
\text { Yearly } \\
\text { value }\end{array}$ \\
\hline 2014 & 0.70 & 0.40 & 1.6555 & 1.5235 & 0.81 & 0.54 & 20.5344 & 23.6395 & 0.83 & 0.38 & 5.5639 & 5.8297 \\
\hline 2015 & 0.55 & 0.33 & 1.1344 & 1.0757 & 0.81 & 0.42 & 16.9935 & 18.1730 & 0.83 & 0.30 & 4.2749 & 4.3274 \\
\hline 2016 & 0.38 & 0.41 & 1.1775 & 1.0970 & 0.77 & 0.63 & 8.7636 & 10.2641 & 0.84 & 0.38 & 2.9436 & 3.1370 \\
\hline
\end{tabular}

$C v$, coefficient of variation; $r$, correlation coefficient.

表4 2014-2016年小时与日尺度优化水分利用效率 $(o W U E)$ 的 $k^{*}$ 值及与 固有水分利用效率 $(u W U E)$ 的相关系数 $(r)$ 比较

Table $4 k^{*}$ values of optimal water use efficiency (oWUE) and its correlation coefficient $(r)$ with $o W U E$ and underlying water use efficiency $(u W U E)$ at the hourly and daily scales during 2014-2016

\begin{tabular}{|c|c|c|c|c|c|c|}
\hline \multirow{3}{*}{$\begin{array}{l}\text { 年份 } \\
\text { Year }\end{array}$} & \multicolumn{2}{|c|}{$k^{*}$ 值 $k^{*}$ value } & \multicolumn{2}{|c|}{$r\left(k=k^{*}\right)$} & \multicolumn{2}{|c|}{$r(k=0.5)$} \\
\hline & \multicolumn{2}{|c|}{ 小时尺度 日尺度 } & \multicolumn{2}{|c|}{ 小时尺度 日尺度 } & \multicolumn{2}{|c|}{ 小时尺度 日尺度 } \\
\hline & Hourly & Daily & Hourly & Daily & Hourly & Daily \\
\hline 2014 & 0.42 & 0.40 & 0.76 & 0.85 & 0.75 & 0.83 \\
\hline 2015 & 0.36 & 0.40 & 0.75 & 0.84 & 0.74 & 0.83 \\
\hline 2016 & 0.38 & 0.37 & 0.82 & 0.85 & 0.80 & 0.84 \\
\hline
\end{tabular}

差 0.1 左右, 但采用 $k^{*}$ 值对相关系数的改进作用有限, 说明优化的水分利用效率指标 $o W U E$ 在描述植被碳 水耦合的内在属性方面与 $u W U E$ 之间没有明显差别。

为进一步分析优化水分利用效率在模拟 $G P P$ 动 态上的表现, 取值 $k^{*}$ 为 0.4 作为 $o W U E$ 的计算参数。

\section{3 日GPP动态过程的模拟}

对黄土高原半干旱草地生态系统2014-2016年 生长季日尺度 $G P P$ 数据进行模拟, 3 年数据趋势一致, 以2015年生长季为例, 模拟结果如图3。年尺度 $W U E 、 I W U E 、 u W U E$ 取值见表3, 年尺度 $o W U E$ 取值 为3.198 2。IWUE、 $u W U E$ 和 $O W U E$ 估测的GPP值在 生长季前期和后期存在明显的高估现象, 而在生长 盛期, $W U E$ 的估算值波动幅度明显偏大。对生长盛 期的实测和估测 $G P P$ 值进行对比分析(图3), $o W U E$ 模拟 $G P P$ 的效果明显好于其他 3 个指标 $(N S E=$ $0.36)$, 而 $u W U E$ 和 $W U E$ 的模拟效果相差不大, $u W U E$ 模拟的 $N S E$ 值为 $0.28, W U E$ 模拟的 $N S E$ 值为 0.25 , $u W U E$ 模拟效果略好。 IWUE的模拟效果差, NSE值 为 -0.97 , 显示 $I W U E$ 并不适用于研究地 $G P P$ 动态过 程的模拟。

\section{3 讨论}

$W U E 、 G P P / E T$ 通常在月到年尺度上表现出相 对一致性, 在日到小时尺度的更短尺度上, $V P D$ 通 过影响气孔导度对碳水耦合过程产生显著影响, 这
是提出 $I W U E$ 和 $u W U E$ 的理论基础。通过引入 $V P D$ 这 一变量, 使得新定义的水分利用效率指标能够在日 甚至小时尺度上表现更为合理，为这一指标用于分 析水分利用效率的日变化, 模拟碳水耦合过程提供 了更好的参数。

通过对黄土高原半干早草地植被观测数据的 分析，不同生态系统水分利用效率指标在日和小时 尺度上, 相关系数的比较结果与Zhou等 $(2014 ， 2015)$ 在美国不同生态系统中的比较结果一致, $u W U E$ 比 $I W U E$ 表现得更好, 表明针对黄土高原半干早草地 植被, $u W U E$ 更适合在日到小时的较小时间尺度进 行碳水耦合特征分析。变异系数的比较结果显示 $u W U E$ 仍然表现最好, 但IWUE比 $W U E$ 要差, 显示了 更大的时间波动性，与 Beer等(2009)和 Keenan等 (2013)的应用结果有差别。这个差别可能是因为研 究区大陆型半干旱季风气候特征导致, 具体原因需 要未来进一步分析探讨。

在年尺度上, $W U E$ 的波动远小于 $I W U E$ 和 $u W U E$ 。这个结果表明在年尺度分析生态系统水分 利用效率时，用 $W U E$ 仍是适宜的，反过来说明 $I W U E$ 和 $u W U E$ 指标适合在短时间尺度上分析植被 碳水耦合特征。

对优化的水分利用效率指标所对应的 $k^{*}$ 的分析 结果显示, 在黄土高原半干旱区, 相对于 $u W U E$, 尽 管 $o W U E$ 相关系数提高有限, 但是 $k^{*}$ 的值已经下降 了 0.1, 对比Zhou等(2015)在不同生态系统之间的比 较分析结果, 0.1 是比较大的差值。Zhou等(2015)认 为用 $u W U E$ 取代优化的水分利用效率指标可以取得 满意效果, 但是我们通过 $k=0.4$ 的 $o W U E$ 指标模拟 $G P P$ 显示, 这个优化系数在模拟 $G P P$ 动态上比 $u W U E$ 具有更高的精度。从模拟 $G P P$ 的应用角度, 我 们认为用 $u W U E$ 取代 $o W U E$ 指标这一结论并不适合 本研究区, $k=0.4$ 的 $o W U E$ 指标可能具有更好的应用 价值。 

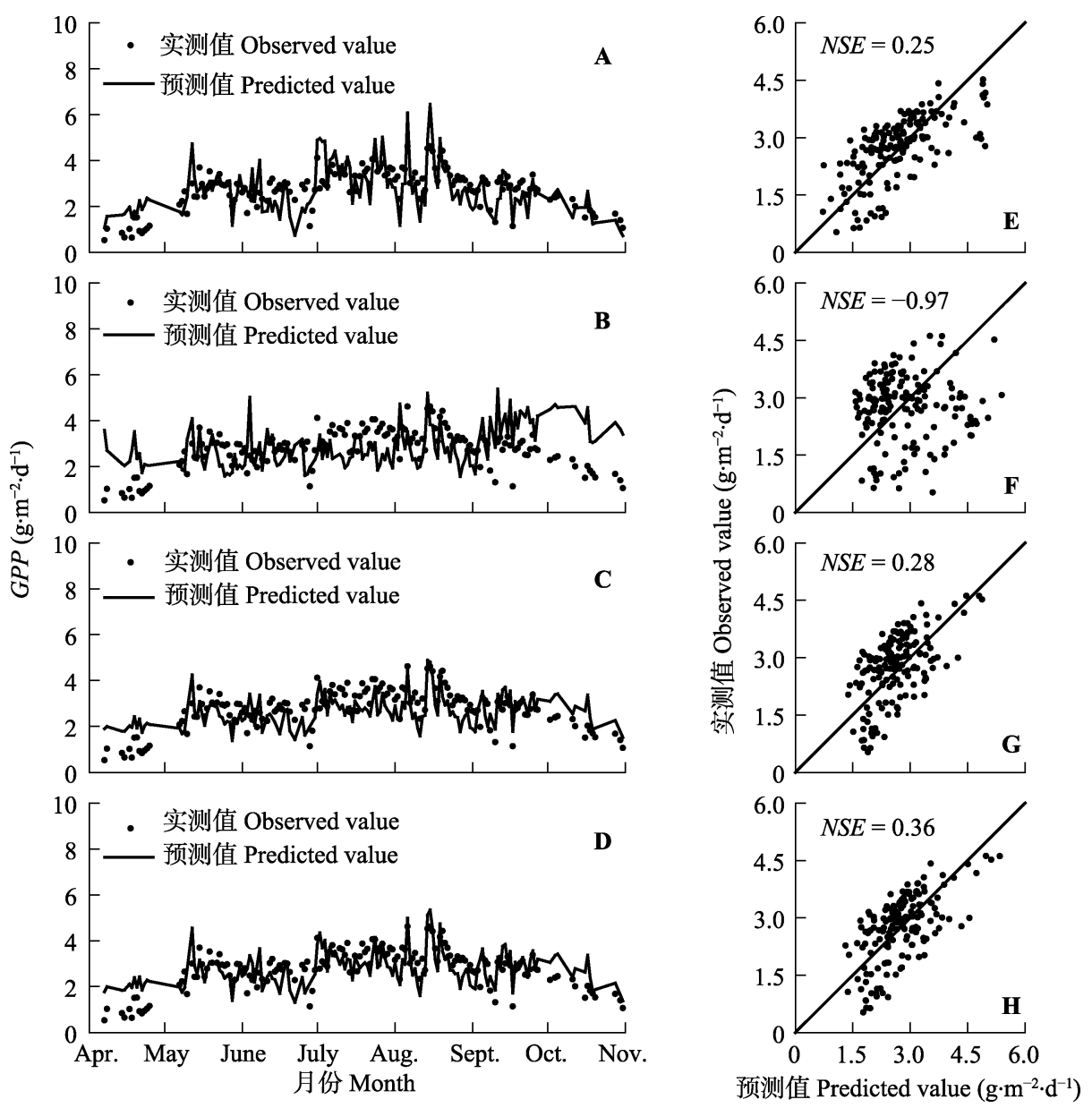

图3 2015年日尺度总初级生产力 $(G P P)$ 实测值与模拟值的比较及其 $N S E$ 值(A和 $\mathbf{E}$ 为水分利用效率, $\mathbf{B}$ 和 $\mathbf{F}$ 为内在水分利用效率, $\mathbf{C}$ 和 $\mathbf{G}$ 为固有水分利用效率, $\mathbf{D}$ 和 $\mathbf{H}$ 为优化水分利用效率)。NSE，纳什效率系数。

Fig. 3 Comparisons between observed and predicted daily gross primary production (GPP) in 2015: A and $\mathbf{E}$ with water use efficiency, $\mathbf{B}$ and $\mathbf{F}$ with inherent water use efficiency, $\mathbf{C}$ and $\mathbf{G}$ with underlying water use efficiency, and $\mathbf{D}$ and $\mathbf{H}$ with optimal water use efficiency. NSE, Nash-Sutcliffe efficiency coefficient.

适合短时间尺度(小时到日)的水分利用效率指 标的一个重要应用领域是将其作为一个参数用于陆 面过程模型中植被碳水耦合过程模拟，或者用于大 尺度 $G P P$ 或 $E T$ 的估算。我们的结果证实 $u W U E$ 和 $o W U E$ 能改进对 $G P P$ 的模拟精度, 但是 $I W U E$ 的模拟 效果很差, 这可能与IWUE的波动幅度大有关。另外, $I W U E 、 u W U E$ 以及 $o W U E$ 在生长期的早期和晚期的 模拟结果均偏大, 而WUE指标在这一阶段模拟效果 较好。导致这一结果的原因可能与生育期早期和晚 期偏低的 $V P D$ (因为气温偏低)有关, 偏低的 $V P D$ 带 来较大的计算误差, 相关的影响机制有待进一步分 析。因此我们建议, 利用 $u W U E$ 和 $o W U E$ 模拟黄土高 原半干旱草地植被 $G P P$ 或 $E T$ 的方法主要应用于植 被生长盛期, 生长季早期和晚期的模拟不建议使用 该指标。

\section{4 结论}

本研究首次比较分析了 $W U E 、 I W U E$ 及 $u W U E$ 在黄土高原干旱区典型草地植被上的应用, 主要结 论如下:

(1) IWUE和 $u W U E$ 适合在日和小时尺度上分析 植被的碳水耦合特征, $u W U E$ 比IWUE 表现更好; $I W U E$ 随时间的波动更大, 稳定性较差, $u W U E$ 的稳 定性最好; WUE适合分析年尺度的碳水耦合特征, 包括年际变化。

(2)一个 $k=0.4$ 的优化水分利用效率指标 $(G P P \times$ $\left.V P D^{0.4} / E T\right)$ 在描述生态系统碳水耦合特征方面与 $u W U E$ 相比并没有本质性改善, 但用在 GPP动态的 模拟效果有明显改善。

(3) $o W U E$ 指标和 $u W U E$ 指标, 相对于 $W U E$ 指标, 
能明显地改善植被生长盛期的 $G P P$ 动态过程模拟, 但在生育期早期和晚期的模拟效果比 $W U E$ 差。 $I W U E$ 指标不适合模拟 $G P P$ 动态过程。

本研究结果对研究全球变化背景下黄土高原 区域气候变化对草地植被碳同化和水分蒸散的影 响、草地生态系统对大气 $\mathrm{CO}_{2}$ 浓度上升的响应, 以及 模拟区域生态系统碳水交换过程有重要的借鉴和指 导意义。

\section{基金项目 国家自然科学基金(41390463)。}

\section{参考文献}

Baldocchi D (1994). A comparative study of mass and energy exchange rates over a closed $\mathrm{C}_{3}$ (wheat) and an open $\mathrm{C}_{4}$ (corn) crop: II. $\mathrm{CO}_{2}$ exchange and water use efficiency. Agricultural and Forest Meteorology, 67(3-4), 291-321.

Battipaglia G, Saurer M, Cherubini P, Calfapietra C, McCarthy $\mathrm{HR}$, Norby RJ, Cotrufo MF (2013). Elevated $\mathrm{CO}_{2}$ increases tree-level intrinsic water use efficiency: Insights from carbon and oxygen isotope analyses in tree rings across three forest FACE sites. The New Phytologist, 197, 544-554.

Beer C, Ciais P, Reichstein M, Baldocchi D, Law BE, Papale D, Soussana JF, Ammann C, Buchmann N, Frank D, Gianelle D, Janssens IA, Knohl A, Kostner B, Moors E, Roupsard O, Verbeeck H, Vesala T, Williams CA, Wohlfahrt G (2009). Temporal and among-site variability of inherent water use efficiency at the ecosystem level. Global Biogeochemical Cycles, 23, GB2018. doi: 10.1029/ $2008 G B 003233$.

Beer C, Reichstein M, Ciais P, Farquhar GD, Papale D (2007). Mean annual GPP of Europe derived from its water balance. Geophysical Research Letters, 34, L05401, doi:10.1029/2006GL029006, 2007.

Farquhar G (1977). Stomatal function in relation to leaf metabolism and environment: Stomatal function in the regulation of gas exchange. Symposia of the Society for Experimental Biology, 31, 471-505.

Grossiord C, Gessler A, Granier A, Pollastrini M, Bussotti F, Bonal D (2014). Interspecific competition influences the response of oak transpiration to increasing drought stress in a mixed Mediterranean forest. Forest Ecology and Management, 318, 54-61.

Hu ZM, Yu GR, Wang QF, Zhao FH (2009). Ecosystem level water use efficiency: A review. Acta Ecologica Sinica, 29, 1498-1507. (in Chinese with English abstract) [胡中民, 于贵瑞, 王秋风, 赵风华 (2009). 生态系统水分利用效 率研究进展. 生态学报, 29, 1498-1507.]

Keenan TF, Hollinger DY, Bohrer G, Dragoni D, Munger JW, Schmid HP, Richardson AD (2013). Increase in forest water-use efficiency as atmospheric carbon dioxide concentrations rise. Nature, 499, 324-327.

Legates DR, McCabe GJ (1999). Evaluating the use of "goodness-of-fit" measures in hydrologic and hydroclimatic model validation. Water Resources Research, 35, 233-241.

Leonardi S, Gentilesca T, Guerrieri R, Ripullone F, Magnani F, Mencuccini M, Noije TV, Borghetti M (2012). Assessing the effects of nitrogen deposition and climate on carbon isotope discrimination and intrinsic water-use efficiency of angiosperm and conifer trees under rising $\mathrm{CO}_{2}$ conditions. Global Chang Biology, 18, 2925-2944.

Niu SL, Xing XR, Zhang Z, Xia JY, Zhou XH, Song B, Li LH, Wan SQ (2011). Water-use efficiency in response to climate change: From leaf to ecosystem in a temperate steppe. Global Change Biology, 17, 1073-1082.

Priestley GHB, Taylor RJ (1972). On the assessment of surface heat flux and evaporation using large-scale parameters. Monthly Weather Review, 100, 81-92.

Scanlon TM, Albertson JD (2004). Canopy scale measurements of $\mathrm{CO}_{2}$ and water vapor exchange along a precipitation gradient in southern Africa. Global Change Biology, 10, 329-341.

Singh JS, Gupta SR (1977). Plant decomposition and soil respiration in terrestrial ecosystems. Botanical Review, 43, 499-528.

Wang SG, He GJ, Liu DS, Wang XQ (2008). Advances in carbon cycle model of forest ecosystem. Science \& Technology Review, 26(9), 72-77. (in Chinese with English abstract) [王绍刚, 何国金, 刘定生, 汪小钦 (2008). 森林碳循环模型方法研究进展. 科技导报, 26(9), 72-77.]

Wohlfahrt G, Haslwanter A, Hortnagl L, Jasoni RL, Fenstermaker LF, Arnone JA, Hammerle A (2009). On the consequences of the energy imbalance for calculating surface conductance to water vapour. Agricultural and Forest Meteorology, 149, 1556-1559.

Yang YT, Long D, Shang SH (2013). Remote estimation of terrestrial evapotranspiration without using meteorological data. Geophysical Research Letters, 40, 3026-3030.

Yu GR, Wang QF et al. (2010). Ecophysiology of Plant Photosynthesis, Transpiration, and Water Use. Science Press, Beijing. (in Chinese) [于贵瑞, 王秋凤等 (2010). 植物光合、蒸腾与水分利用的生理生态学. 科学出版社, 北京.]

Zhang LX, Hu ZM, Fan JW, Shao QQ, Tang FP (2014). Advances in the spatiotemporal dynamics in ecosystem water use efficiency at regional scale. Advances in Earth Science, 29, 691-699. (in Chinese with English abstract) [张良侠, 胡中民, 焚江文, 邵全琴, 唐风沛 (2014). 区 域尺度生态系统水分利用效率的时空变异特征研究进 展. 地球科学进展, 29, 691-699.]

Zhao C, Yuan GF, Liu X, Shao MA, Yi XB (2015). Application 
of cosmic-ray method to soil moisture measurement of grassland in the Loess Plateau. Acta Pedologica Sinica, 52, 1438-1444. (in Chinese with English abstract) [赵纯, 袁 国富, 刘晓, 邵明安, 易小波 (2015). 宇宙射线土壤水 分观测方法在黄土高原草地植被的应用. 土壤学报, 52, 1438-1444.]

Zhou S, Yu B, Huang Y, Wang G (2014). The effect of vapor pressure deficit on water use efficiency at the subdaily time scale. Geophysical Research Letters, 41, 5005-5013.

Zhou S, Yu B, Huang Y, Wang G (2015). Daily underlying water use efficiency for AmeriFlux sites. Journal of Geophysical Research Biogeosciences, 120, 887-902.

责任编委: 赵 平 责任编辑: 王 葳

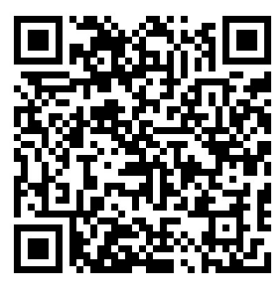

扫码向作者提问

\section{《气候变暖下大兴安岭落叶松径向生长对温度的响应》作者更正}

2017年3月 《植物生态学报》第41卷第3期刊出的研究论文《气候变暖下大兴安岭落叶松径向生长对温 度的响应》第282页的胸高断面积增量 $(B A I)$ 计算公式 $B A I=0.25\left[D B H^{2}+(D B H-2 a)^{2}\right]$ 更正为:

$$
B A I=0.25 \pi\left[D B H^{2}-(D B H-2 a)^{2}\right]
$$

由于本人疏忽, 给读者带来不便, 敬请谅解! 在此, 谨代表本文全体作者向广大读者致以真诚的歉意!

$$
\text { 常永兴 于2017年6月 }
$$

